\title{
Sentinel lymph node mapping with indocyanine green in cervical cancer patients undergoing open radical hysterectomy: a single-institution series
}

\author{
Nicolò Bizzarri ${ }^{1}$ (1) Pedone Anchora Luigi ${ }^{1}$. Gabriella Ferrandina ${ }^{1,2}$. Gian Franco Zannoni ${ }^{2,3}$. \\ Maria Vittoria Carbone ${ }^{1}$. Camilla Fedele $^{1}$. Elena Teodorico ${ }^{1}$. Valerio Gallotta ${ }^{1}$. Salvatore Gueli Alletti ${ }^{1}$. \\ Vito Chiantera ${ }^{4} \cdot$ Anna Fagotti $^{1,2} \cdot$ Giovanni Scambia $^{1,2}$ (D) Francesco Fanfani ${ }^{1,2}$
}

Received: 9 August 2020 / Accepted: 10 September 2020 / Published online: 30 September 2020

(c) The Author(s) 2020

\begin{abstract}
Purpose To assess the rate of bilateral sentinel lymph node (SLN) detection with indocyanine green (ICG), to evaluate the sensitivity and the negative predictive value of cervical cancer patients undergoing open radical hysterectomy; to compare open versus minimally invasive SLN biopsy performance and to assess factors related to no/unilateral SLN mapping.

Methods We retrospectively reviewed consecutive patients with FIGO 2018 stage IA1 with lymph-vascular space involvement to IIB and IIIC1p cervical carcinoma who underwent SLN mapping with ICG followed by systematic pelvic lymphadenectomy between 05/2017 and 06/2020. Patients were divided according to surgical approach for statistical analysis. Results Eighty-five patients met inclusion criteria. Twenty-seven (31.8\%) underwent open and 58 (68.2\%) underwent minimally invasive SLN mapping. No difference in any SLN mapping (laparotomy $92.6 \%$ and minimally invasive $91.4 \%$ ) or in SLN bilateral detection (laparotomy $72.0 \%$ and minimally invasive $84.9 \%)(p=0.850$ and $p=0.222$, respectively), in median number of SLNs mapped and retrieved ( 2 in both groups, $p=0.165$ ) and in site of SLN mapping per hemi-pelvis (right side, $p=0273$ and left side, $p=0.618$ ) was evident between open and minimally invasive approach. Per-patient sensitivity of SLN biopsy in laparotomy was $83.3 \%$ (95\% CI 35.9-99.6\%) and the negative predictive value was $95.0 \%$ (95\% CI 76.0-99.1\%). No difference in per-patient sensitivity was noted between two approaches $(p=0.300)$. None of the analyzed variables was associated with no/unilateral SLN mapping.

Conclusion The use of ICG to detect SLN in cervical cancer treated with open surgery allows a bilateral detection, sensitivity and negative predictive value comparable to minimally invasive surgery with potential advantages of ICG compared to other tracers.
\end{abstract}

Keywords Cervical cancer $\cdot$ Sentinel lymph node $\cdot$ Laparotomy $\cdot$ Minimally invasive surgery $\cdot$ Indocyanine green $\cdot$ Detection rate

Electronic supplementary material The online version of this article (https://doi.org/10.1007/s00432-020-03393-6) contains supplementary material, which is available to authorized users.

Giovanni Scambia

giovanni.scambia@policlinicogemelli.it

1 UOC Ginecologia Oncologica, Dipartimento per la salute della Donna e del Bambino e della Salute Pubblica,

Fondazione Policlinico Universitario A. Gemelli, IRCCS, Largo Agostino Gemelli 8, 00168 Rome, Italy

2 Università Cattolica del Sacro Cuore, Largo Francesco Vito 1, 00168 Rome, Italy
3 Unità di Gineco-Patologia e Patologia Mammaria, Dipartimento per la salute della Donna e del Bambino e della Salute Pubblica, Fondazione Policlinico Universitario A. Gemelli, IRCCS, Largo Agostino Gemelli 8, 00168 Rome, Italy

4 Department of Gynecologic Oncology, ARNAS Ospedali Civico di Cristina Benfratelli, University of Palermo, Piazza Nicola Leotta 4/A, 90127 Palermo, Italy 


\section{Introduction}

Despite the introduction of screening and vaccination has significantly reduced the incidence of cervical cancer in developed countries (Brisson 2020; Wright 2015), this tumor still represents the fourth most common cancer worldwide, with 569,847 new cases and 311,365 deaths in 2018 (Arbyn 2020). Treatment of early-stage disease (clinical International Federation of Gynecology and Obstetrics (FIGO) stage IA1-IB1 and IIA1) is represented by radical hysterectomy and pelvic lymphadenectomy with sentinel lymph node (SLN) biopsy (Cibula 2018). After the publication of a randomized controlled trial, which demonstrated an inferior survival outcome if patients with early-stage cervical cancer were treated with minimally invasive, compared to open radical hysterectomy (Ramirez 2018), multiple retrospective studies showed a survival difference between the two approaches, with consequent recommendations amendment by international societies (Koh 2019; Nitecki 2020; Querleu 2020), particularly for tumors larger than $2 \mathrm{~cm}$ (Pedone Anchora 2020).

SLN biopsy has been largely studied in cervical cancer and multiple evidences support its use in early-stage disease treated with primary surgery (Cibula 2018,2019); also, few studies reported the use of SLN biopsy after neo-adjuvant chemotherapy (NACT) (Slama 2012; Robova 2014). Different studies reported the adoption of SLN in open radical surgery with blue dye or radioactive tracer (Silva 2005; Wydra 2006; Altgassen 2007; Kara 2008; Dostalek 2020), but experience on the use of indocyanine green (ICG) in open surgery, is limited to few case reports or case series (Furukawa 2010; Crane 2011; van der Vorst 2011; Schaafsma 2012; Buda 2016; Rychlik 2016). On the other hand, it seems well established that ICG has been showed to be superior to blue dye or radioactive tracer in SLN bilateral detection rate in minimally invasive surgery (Di Martino 2017).

The primary objective of the present study was to assess the rate of bilateral SLN detection with ICG in cervical cancer patients undergoing open radical hysterectomy. Secondary objectives were to evaluate the sensitivity and the negative predictive value of open ICG SLN biopsy, to compare open versus minimally invasive SLN biopsy performance and to assess factors related to no/unilateral SLN mapping.

\section{Methods}

\section{Inclusion and exclusion criteria}

The present retrospective observational cohort study was approved by Institutional Review Board (Dipartimento per la salute della Donna e del Bambino e della Salute Pubblica, number DIPUSVSP-26-05-2059, date 26/05/2020). After signing consent form, consecutive patients who underwent surgery at Fondazione Policlinico A. Gemelli IRCCS (Rome, Italy), for International Federation of Gynecology and Obstetrics (FIGO) 2018 (Bhatla 2018) stage IA1 with lymph-vascular space involvement (LVSI) to IIB and IIIC1p cervical carcinoma between 05/2017 and 06/2020 and had SLN mapping attempt with systematic pelvic lymphadenectomy, were included. Data were retrieved from institution's electronic database. All patients had pre-operative histology confirmation of cervical cancer and underwent pre-operative abdominal Magnetic Resonance Imaging (MRI) scan, pelvic ultrasound scan and chest X-ray. Patients with FIGO stage > IB 1 underwent whole body positron emission tomography (PET)/computed tomography (CT) scan to exclude distant metastasis. Radicality of hysterectomy was classified according to Querleu-Morrow classification (Querleu 2017). Examination under anesthesia was performed at the same time of the surgery. Only women with no evidence of enlarged (i.e., short axis $>10 \mathrm{~mm}$ ) pelvic and para-aortic lymph nodes at pre-operative MRI-scan were submitted to SLN mapping. Patients with pre-invasive cervical disease, who did not undergo systematic pelvic lymphadenectomy, with special histology sub-types (other than squamous cell carcinoma-SCC, adenocarcinoma or adenosquamous carcinoma), allergy to iodine or patients with SLN analyzed with standard H\&E only, were excluded.

Surgical approach was performed by minimally invasive surgery (laparoscopic or robotic) at surgeon's discretion until October 2018 (Ramirez 2018) and for tumors $<2 \mathrm{~cm}$ in compliance with European Society of Gynaecological Oncology (ESGO) statement and National Comprehensive Cancer Network (NCCN) guidelines, using protecting maneuvers (no uterine manipulator and vaginal cuff formation in patients with tumor completely excised at preoperative conization), after that date (Koh 2019; Querleu 2020). Laparotomy approach was performed at surgeon's discretion until October 2018 (Ramirez 2018) and for tumors $\geq 2 \mathrm{~cm}$ after that date. Uterine surgery involved radical hysterectomy in patients who did not wish to retain fertility; and cervical conization or cervical biopsy followed by NACT [as part of a previously described clinical trial, NCT02323841-in press Fertility and Sterility (ClinicalTrials.gov Identifier: NCT02323841) (Clinicaltrial 2014)], in patients who desired to preserve fertility with tumor diameter $\leq 2 \mathrm{~cm}$ and $>2 \mathrm{~cm}$, respectively. Patients with clinical diagnosis of FIGO stage IIB, underwent NACT followed by radical hysterectomy. All patients undergoing open surgery were treated with radical hysterectomy. 
FIGO stage refers to pathologic stage for patients who underwent primary surgery and to clinical stage at diagnosis for patients undergoing NACT.

\section{Sentinel lymph node assessment}

Technique and method for SLN mapping was the same in all cases. SLN was detected after $1 \mathrm{ml}$ superficial and deep cervical injections of ICG (diluted with sterile water at $1.25 \mathrm{mg}$ / $\mathrm{ml}$ ) at 3 and 9 o'clock. About 15 min after cervical injection, pelvic retroperitoneal space was opened, and lymph nodes were assessed with near infra-red (NIR) camera: Visera Elite II (Olympus, Tokyo, Japan) and Pinpoint (Stryker, Kalamazoo, Michigan, US) in case of laparoscopic or Da Vinci $\mathrm{Xi}$ (Intuitive, Sunnyvale, California, US) in case of robotic approach; SLN in open surgery was detected with SPY Portable Handheld Imager (SPY-PHI) camber (Stryker, Kalamazoo, Michigan, US). SLN was defined as the ICG-positive pelvic node closest to the tumor; retroperitoneal spaces were explored with the following order to assess SLN presence: external iliac, interiliac, obturator, common iliac, parametrial and pre-sacral and low para-aortic area (Marnitz 2006; Balaya 2019). All mapped SLNs were removed and analyzed with ultrastaging (Cibula 2012) or with one-step nucleic acid amplification (OSNA) (Bizzarri 2020).

Ultrastaging procedure was performed as follows: hematoxylin and Eosin (H\&E) negative nodes were entirely sectioned at $150 \mu \mathrm{m}$ intervals, until exhaustion of the lymph node, according to the length of the long axis. Each level was examined by H\&E and AE1/AE3. Low volume disease was defined by the American Joint Committee on Cancer (AJCC) (Schwartz 2002): macro-metastases were defined as cancer deposits larger than $2.0 \mathrm{~mm}$; micro-metastases were defined as deposits between 0.2 and $2.0 \mathrm{~mm}$; and isolated tumor cells were defined as deposits no greater than $0.2 \mathrm{~mm}$, including the presence of single non-cohesive cytokeratinpositive cancer cells.

OSNA analysis of SLNs was performed as per previously described protocol.

Systematic bilateral pelvic lymphadenectomy was performed in all patients, regardless SLN mapping. Non-SLNs were examined with routine $\mathrm{H} \& \mathrm{E}$ staining.

\section{Statistical analysis}

Standard descriptive statistics were used to evaluate the distribution of each variable. Continuous variables were reported as median and categorical variables as frequency and percentage. Comparison of each variable between the groups of patients have been performed with $t$ test for continuous variables and Chi-square or Fisher's exact test for categorical variables. Sensitivity, negative predictive value, and false-negative rates were calculated per patient and per hemi-pelvis. We considered findings to be false negative when lymphatic mapping showed drainage to one or more SLNs in a hemi-pelvis, biopsy of the SLN(s) revealed no metastases, and the patient had at least one metastatic non-SLN. A positive non-SLN in a hemipelvis with no SLN identified on lymphatic mapping was not considered to indicate a false-negative finding as our practice was to perform a complete pelvic lymphadenectomy in any case. All $p$ values reported were two sided and a $p$ value $<0.05$ was considered statistically significant. Analysis was computed using SPSS version 26.0 (IBM Corporation 2018, Armonk, NY: IBM Corp.).

\section{Results}

\section{Entire cohort characteristics}

Eighty-five patients met inclusion criteria and underwent ICG injection to detect SLN. Twenty-seven (31.8\%) underwent open and $58(68.2 \%)$ underwent minimally invasive SLN mapping. Of these, $43(50.6 \%)$ underwent laparoscopic and $15(17.6 \%)$ robotic approach.

Table 1 shows characteristics of the entire cohort. Nine $(10.6 \%)$ patients were submitted to NACT before radical hysterectomy. Most of patients had SCC histology $(56.5 \%)$, grade $2(51.8 \%)$, maximum tumor diameter $<4 \mathrm{~cm}(56.5 \%)$ and underwent type $\mathrm{B}$ or $\mathrm{C}$ radical hysterectomy $(82.4 \%)$. Overall, $63(74.1 \%)$ patients had bilateral SLN mapping, while $15(17.6 \%)$ and $7(8.2 \%)$ had unilateral and no mapping, respectively. One-hundred and seventy-six SLNs were detected and retrieved. Median number of SLNs removed was 2 (range, 1-5) per patient. The most frequent site of SLN mapping was external iliac in $76(58.5 \%)$, followed by obturator in $38(29.2 \%)$, internal iliac in $7(5.4 \%)$ and common iliac in $5(3.8 \%)$ cases. Two (1.5\%) and two (1.5\%) patients had para-aortic and pre-sacral mapping, respectively, but none of these was isolated. No patient presented allergic reaction to ICG in the entire cohort.

Overall, $20(23.5 \%)$ patients had metastatic lymph nodes, and $18(21.2 \%)$ had metastatic SLN. Of these, 3 (3.5\%) had ITCs, $13(15.3 \%)$ had micro- and $2(2.4 \%)$ had macro-metastases.

In the entire cohort, when calculated per-patient, the sensitivity of SLN biopsy was $90.0 \%$ (95\% CI 68.3-98.8\%) and the negative predictive value was $96.7 \%$ (95\% CI 88.6-99.1\%). When calculated per hemi-pelvis, the sensitivity of SLN biopsy was $97.1 \%$ (95\% CI 85.1-99.9\%) and the negative predictive value was $99.1 \%$ (95\% CI 93.9-99.9\%) (Supplementary Table 1). 
Table 1 Patients' characteristics

\begin{tabular}{|c|c|}
\hline Characteristic & $N=85$, median (range, $\%)$ \\
\hline Age (years) & $44(27-80)$ \\
\hline BMI (kg/m2) & $23.0(17.3-36.0)$ \\
\hline \multicolumn{2}{|l|}{ Neo-adjuvant chemotherapy } \\
\hline No & $76(89.4)$ \\
\hline Yes & $9(10.6)$ \\
\hline \multicolumn{2}{|l|}{ Pre-operative conization } \\
\hline No & $58(68.2)$ \\
\hline Yes & $27(31.8)$ \\
\hline \multicolumn{2}{|l|}{ Approach } \\
\hline Laparoscopy & $43(50.6)$ \\
\hline Robot & $15(17.6)$ \\
\hline Laparotomy & $27(31.8)$ \\
\hline \multicolumn{2}{|c|}{ Type of surgery on uterus/cervix at time of SLN biopsy } \\
\hline Conization & $7(8.2)$ \\
\hline Biopsy only & $2(2.4)$ \\
\hline Type A RH & $6(7.1)$ \\
\hline Type B RH & $31(36.5)$ \\
\hline Type C RH & $39(45.9)$ \\
\hline \multicolumn{2}{|l|}{ SLN mapping } \\
\hline No & $7(8.2)$ \\
\hline Unilateral & $15(17.6)$ \\
\hline Bilateral & $63(74.1)$ \\
\hline \multicolumn{2}{|l|}{ SLN analysis } \\
\hline No mapping & $7(8.2)$ \\
\hline Ultra staging & $49(57.6)$ \\
\hline OSNA & $29(34.1)$ \\
\hline Median number SLN & $2(1-5)$ \\
\hline \multicolumn{2}{|l|}{ Histology } \\
\hline $\mathrm{SCC}$ & $48(56.5)$ \\
\hline Adenocarcinoma & $34(40.0)$ \\
\hline Adenosquamous & $3(3.5)$ \\
\hline \multicolumn{2}{|l|}{ Grade } \\
\hline 1 & $9(10.6)$ \\
\hline 2 & $44(51.8)$ \\
\hline 3 & $21(87.1)$ \\
\hline Unknown & $11(12.9)$ \\
\hline \multicolumn{2}{|l|}{ LVSI } \\
\hline Negative & $45(52.9)$ \\
\hline Positive & $35(41.2)$ \\
\hline Unknown & $5(5.9)$ \\
\hline Depth of stromal infiltration & $5(0.5-25)$ \\
\hline Maximum tumor diameter (mm) & $20(1-65)$ \\
\hline \multicolumn{2}{|l|}{ Tumor diameter } \\
\hline$\leq 20 \mathrm{~mm}$ & $45(52.9)$ \\
\hline$>20 \mathrm{~mm}$ & $40(47.1)$ \\
\hline \multicolumn{2}{|l|}{ FIGO stage 2018} \\
\hline IA1 & $7(8.2)$ \\
\hline IA2 & $4(4.7)$ \\
\hline IB1 & $19(22.4)$ \\
\hline IB2 & $20(23.5)$ \\
\hline
\end{tabular}

Table 1 (continued)

\begin{tabular}{ll}
\hline Characteristic & $N=85$, median (range, \%) \\
\hline IB3 & $6(7.1)$ \\
IIA1 & $4(4.7)$ \\
IIB & $5(5.9)$ \\
IIIC1p & $20(23.5)$ \\
Number or removed lymph nodes & $18(5-86)$ \\
SLN largest dimension metastasis & \\
No mapping & $7(8.2)$ \\
No & $60(70.6)$ \\
ITC & $3(3.5)$ \\
Micro & $13(15.3)$ \\
Macro & $2(2.4)$ \\
\hline
\end{tabular}

$B M I$ body mass index, $S L N$ sentinel lymph node, OSNA one-step nucleic acid amplification, SCC squamous cell carcinoma, LVSI lymph-vascular space involvement, FIGO international federation of gynecology and obstetrics

\section{Comparison of open and minimally invasive approach}

Comparison of characteristics of minimally invasive (58, $68.2 \%)$ and open approach $(27,31.8 \%)$ is reported in Table 2. Patients who underwent open approach reported higher rate of grade $3(p=0.028)$ and positive LVSI $(p=0.003)$, deeper stromal infiltration (median $8 \mathrm{~mm}$ vs. $5 \mathrm{~mm}, p=0.016$ ), larger maximum tumor diameter (median $25 \mathrm{~mm}$ vs. $15 \mathrm{~mm}, p=0.003$ ) and higher FIGO stage $(p=0.039)$. Despite these differences, no difference in any SLN mapping or in SLN bilateral detection was evident between open and minimally invasive approach ( $p=0.850$ and $p=0.222$, respectively). Moreover, there was no difference in median number of SLNs mapped and retrieved between the two approaches ( 2 in both groups, $p=0.165)$ and in site of SLN mapping per hemi-pelvis (right side, $p=0273$ and left side, $p=0.618$ ). Figures 1 and 2 show two examples of SLN mapping in open and minimally invasive surgery, respectively.

In the sub-group of laparotomy patients, per-patient sensitivity of SLN biopsy was 83.3\% (95\% CI 35.9-99.6\%) and negative predictive value was $95.0 \%$ (95\% CI 76.0-99.1\%). When calculated by hemi-pelvis, the sensitivity of SLN biopsy was $88.9 \%$ (95\% CI 51.7-99.7\%) and the negative predictive value was 97.1\% (95\% CI 84.3-99.5\%) (Supplementary Table 1). For what concerns minimally invasive group, the sensitivity of SLN biopsy was $92.9 \%$ (95\% CI $66.1-99.8 \%$ ) and the negative predictive value was $97.5 \%$ (95\% CI 85.5-99.6\%) in the per-patients and they were $100.0 \%$ (95\% CI $86.8-100.0 \%$ ) and $100.0 \%$ respectively, in the per-hemipelvis calculation (Supplementary Table 1). No difference in per-patient sensitivity was noted between laparotomy and minimally invasive surgery $(p=0.300)$. 
Table 2 Comparison of characteristics of patients undergoing SLN mapping by laparotomy (LPT) and minimally invasive surgery (MIS)

\begin{tabular}{|c|c|c|c|}
\hline Characteristic & $\mathrm{LPT}=27, \%$ & $\mathrm{MIS}=58, \%$ & $p$ value \\
\hline Age (years) & $45(27-80)$ & $34.5(29-77)$ & 0.421 \\
\hline BMI (kg/m2) & $23.0(18.7-35.0)$ & $23.3(17.3-36.0)$ & 0.718 \\
\hline \multicolumn{4}{|l|}{ Neo-adjuvant chemotherapy } \\
\hline No & $23(85.2)$ & $53(91.4)$ & 0.456 \\
\hline Yes & $4(14.8)$ & $5(8.6)$ & \\
\hline Histology & & & 0.483 \\
\hline SCC & $16(59.3)$ & $32(55.2)$ & \\
\hline Adenocarcinoma & $11(40.7)$ & $23(39.7)$ & \\
\hline Adenosquamous & 0 & $3(5.2)$ & \\
\hline Grade $^{\mathrm{a}}$ & & & 0.028 \\
\hline 1 & $2(7.4)$ & $7(12.1)$ & \\
\hline 2 & $11(40.7)$ & $33(56.9)$ & \\
\hline 3 & $12(44.4)$ & $9(15.5)$ & \\
\hline Unknown & $2(7.4)$ & $9(15.5)$ & \\
\hline LVSI $^{\mathrm{a}}$ & & & 0.003 \\
\hline Negative & $8(29.6)$ & $37(63.8)$ & \\
\hline Positive & $17(63.0)$ & $18(31.0)$ & \\
\hline Unknown & $2(7.4)$ & $3(5.2)$ & \\
\hline Depth of stromal infiltration & $8(0.4-25)$ & $5(0.6-17)$ & 0.016 \\
\hline Maximum tumor diameter (mm) & $25(5-65)$ & $15(1-50)$ & 0.003 \\
\hline Tumor diameter & & & 0.005 \\
\hline$\leq 20 \mathrm{~mm}$ & $8(29.6)$ & $37(63.8)$ & \\
\hline$>20 \mathrm{~mm}$ & $19(70.4)$ & $21(36.2)$ & \\
\hline FIGO stage 2018 & & & 0.039 \\
\hline IA1 & 0 & $7(12.1)$ & \\
\hline IA2 & 0 & $4(6.9)$ & \\
\hline IB1 & $5(18.5)$ & $14(24.1)$ & \\
\hline IB2 & 7 (25.9) & $13(22.4)$ & \\
\hline IB3 & $5(18.5)$ & $1(1.7)$ & \\
\hline IIA1 & $1(3.7)$ & $3(5.2)$ & \\
\hline IIB & $3(11.1)$ & $2(3.4)$ & \\
\hline IIIC1p & $6(22.2)$ & $14(24.1)$ & \\
\hline SLN mapping & & & 0.850 \\
\hline No & $2(7.4)$ & $5(8.6)$ & \\
\hline Yes & $25(92.6)$ & $53(91.4)$ & \\
\hline SLN detection ${ }^{\mathrm{b}}$ & & & 0.222 \\
\hline Unilateral & $7(28.0)$ & $8(15.1)$ & \\
\hline Bilateral & $18(72.0)$ & $45(84.9)$ & \\
\hline SLN analysis ${ }^{b}$ & & & 0.133 \\
\hline Ultrastaging & $19(76.0)$ & $30(56.6)$ & \\
\hline OSNA & $6(24.0)$ & $23(43.4)$ & \\
\hline Number of $\mathrm{SLN}^{\mathrm{b}}$ & & & 0.476 \\
\hline 1 & $5(20.0)$ & $7(13.2)$ & \\
\hline 2 & $17(68.0)$ & $30(56.6)$ & \\
\hline 3 & $1(4.0)$ & $9(17.0)$ & \\
\hline 4 & $1(4.0)$ & $5(9.4)$ & \\
\hline 5 & $1(4.0)$ & $1(1.9)$ & \\
\hline 6 & 0 & $1(1.9)$ & \\
\hline Median number of SLN & $2(1-5)$ & $2(1-6)$ & 0.165 \\
\hline Median number of removed lymph nodes & $23(8-57)$ & $18(5-86)$ & 0.197 \\
\hline Site of mapping of first Right $\mathrm{SLN}^{\mathrm{c}}$ & & & 0.273 \\
\hline
\end{tabular}


Table 2 (continued)

\begin{tabular}{llll}
\hline Characteristic & LPT $=27, \%$ & MIS $=58, \%$ & $p$ value \\
\hline Obturator & $6(26.1)$ & $13(31.0)$ & \\
Internal iliac & 0 & $4(9.5)$ & $21(50.0)$ \\
External iliac & $16(69.6)$ & $3(7.1)$ & \\
Common iliac & 0 & $1(2.4)$ & \\
Para-aortic & $1(4.3)$ & $13(31.0)$ \\
Site of mapping of first Left SLN & \\
Obturator & $6(30.0)$ & $3(7.1)$ \\
Internal iliac & 0 & $25(59.5)$ \\
External iliac & $13(65.0)$ & $1(2.4)$ \\
Common iliac & $1(5.0)$ & \\
SLN metastasis & & $40(75.5)$ \\
No & $20(80.0)$ & $2(3.8)$ \\
ITC & $1(4.0)$ & $9(17.0)$ & 0.801 \\
Micro & $4(16.0)$ & $2(3.8)$ & \\
Macro & 0 &
\end{tabular}

Bold significance values are $p<0.05$

$B M I$ body mass index, SCC squamous cell carcinoma, LVSI lymph-vascular space involvement, FIGO international federation of gynecology and obstetrics, SLN sentinel lymph node, OSNA one-step nucleic acid amplification, ITC isolated tumor cell

a Analysis performed on cases with available data only

${ }^{\mathrm{b}}$ Analysis performed on 78 cases with uni/bilateral mapping

${ }^{c}$ analysis performed on 65 right hemipelvis mapping, 7 patients had pelvic mapping but site not specified; total number of SLNs retrieved: 176

${ }^{\mathrm{d}}$ analysis performed on 62 left hemipelvis mapping, 7 patients had pelvic mapping but site not specified; total number of SLNs retrieved: 176
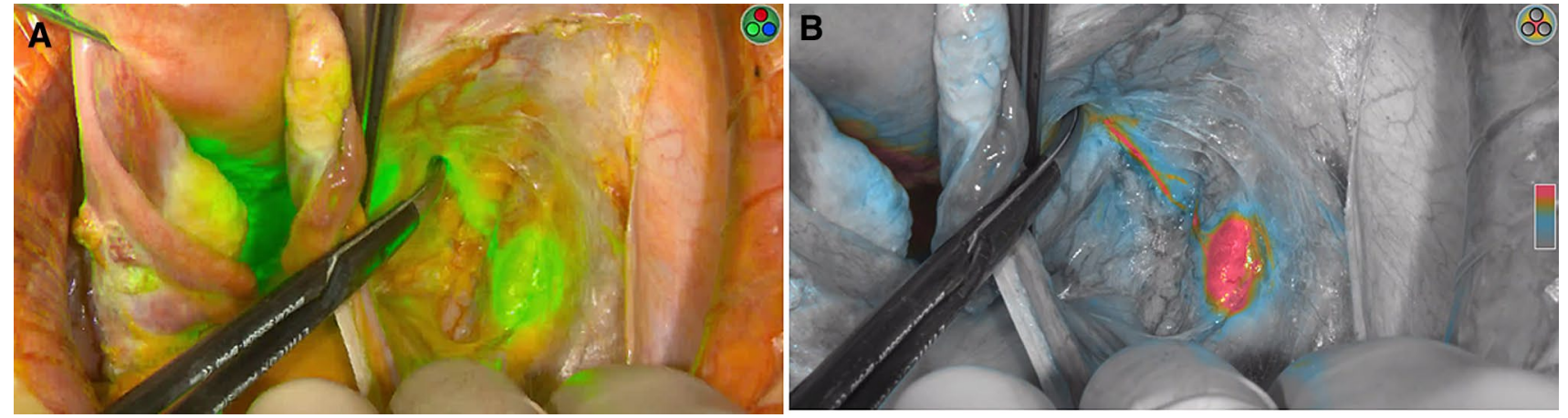

Fig. 1 Right external iliac SLN detected with ICG green mode (1a) and color-segmented fluorescence (1b) during open surgery

\section{Comparison of no/unilateral and bilateral SLN detection}

Analysis of variables associated with bilateral SLN detection, compared with no/unilateral SLN detection within the entire cohort, is reported in Table 3. None of the analyzed characteristics was significantly different between the two groups. In particular, no surgery-related, or tumor-related variable was associated with higher bilateral SLN mapping. When we divided the study period in two parts, considering the first as "learning period" (33 patients, until 12/2018) and the second "experienced period" (52 patients, from 01/2019), no difference was observed in bilateral SLN detection rate $(p=0.450)$.

\section{Discussion}

The aim of this study was to compare the bilateral SLN detection rate with ICG of patients undergoing open and minimally invasive radical hysterectomy for cervical cancer. Following the publication of the LACC trial (Ramirez 


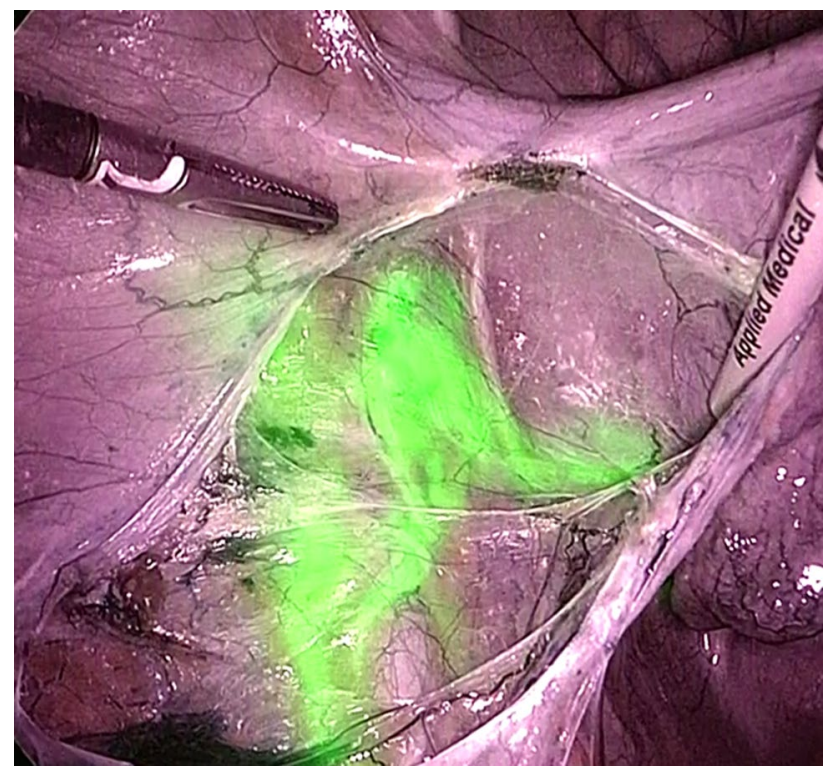

Fig. 2 Left external iliac SLN detected with ICG during laparoscopic surgery

2018), which demonstrated survival advantage in patients operated with laparotomy approach, international societies have changed their recommendations in terms of surgical approach for radical hysterectomy in early-stage cervical cancer (Koh 2019, Querleu 2020). Multiple subsequent observational studies have confirmed LACC trial results, particularly for patients with tumors larger than $2 \mathrm{~cm}$ (Nitecki 2020, Pedone Anchora 2020). SLN has become essential part of the operative management of early-stage cervical cancer, as it provides important information on lymph nodes at highest probability of metastasis and it allows a thorough analysis of these nodes by ultrastaging (Cibula 2012) or OSNA (Bizzarri 2020). Such analysis allows to detect low-volume metastases, which represents an important information, having some authors described the negative prognostic impact of micrometastases (Kocian 2020); nevertheless, not all studies support the negative impact of micrometastases in cervical cancer (Guani 2020). Moreover, ICG has been demonstrated to be superior to other tracers alone (such as blue dye or radioactive tracers), and comparable with combination of blue and radioactive tracer in detecting SLNs in cervical cancer (Di Martino 2017). With this background, we intended to assess whether ICG provides such a high bilateral SLN detection rate in open surgery as in minimally invasive surgery, in view of the clinical practice change that many centers across the world had with regards to the surgical approach.

With our results, we showed no difference in any SLN and bilateral SLN detection between patients undergoing SLN mapping with open surgery compared to laparoscopic or robotic surgery. Moreover, with an overall and bilateral detection rate of $92.6 \%$ and $72.0 \%$, SLN mapping with ICG in laparotomy is in line with previously reported pooled data from a diagnostic review, showing overall and bilateral detection rate of $91 \%$ and $60 \%$, respectively (Tax 2015).

Differences in tumor characteristics between patients undergoing open and minimally invasive approach is likely to be related to the approach selection. Even though not significant, the difference in bilateral detection rate between the two approaches $(72.0 \%$ in open versus $84.9 \%$ in minimally invasive), could be explained by the tumor characteristics of patients undergoing open approach. In fact, as previously described by (Dostálek 2018), bilateral SLN detection in large volume cervical cancer was lower, compared to smaller tumors.

Regarding sensitivity and negative predictive value in the per-patient analysis, our results demonstrated that there was no significant difference between patients undergoing open ICG SLN (83.3-95.0\%, respectively) and minimally invasive ICG SLN (92.9-97.5\%, respectively). The same applies for the per-hemipelvis analysis. When compared with other literature studies, we found sensitivity from open ICG SLN in the present series to be slightly lower compared with other sensitivities reported in the literature (ranging from 91.0 to $96.4 \%$ ), with a negative predictive value concordant with other series (ranging from 91.0 to 100\%) (Cibula 2012; Tax 2015; Salvo 2017).

This is not the first study reporting the use of ICG to detect SLN in open surgery for cervical cancer. However, to date, only few case reports or small series were reported (Furukawa 2010; Crane 2011; van der Vorst 2011; Schaafsma 2012; Buda 2016; Rychlik 2016; Snyman 2018; Bedyńska 2019). Table 4 shows the characteristics of the studies which reported on the use of ICG to detect SLN in laparotomy. On the other hand, SLN in open surgery has been largely described with the use of other tracers such as blue dye, radioactive tracer (technetium) or combined technique (Silva 2005; Wydra 2006; Altgassen 2007; Kara 2008; Dostalek 2020). Nevertheless, ICG shows some advantages compared to other tracers: the low-risk of allergic reaction compared to blue dye (Papadia 2017); possibility of performing SLN biopsy in one-step at the time of surgery and the absence of patients exposure to ionizing radiation compared to radioactive tracer; lastly, it allows a real-time visualization of lymphatic channels.

The main limitation of the present study is the possible selection bias for patients submitted to open surgery; on the other hand, we have to acknowledge that this is the first study reporting the use of ICG in open surgery in a relatively large number of patients with cervical cancer, with a novel near infrared technology. 
Table 3 Factors associated with bilateral detection in the entire cohort

\begin{tabular}{|c|c|c|c|}
\hline Characteristic & $\begin{array}{l}\text { No/unilateral mapping } \\
(N=22)\end{array}$ & $\begin{array}{l}\text { Bilateral mapping } \\
(N=63)\end{array}$ & $p$ value \\
\hline Age (years) & $48(29-80)$ & $42(27-75)$ & 0.121 \\
\hline BMI (kg/m2) & $24.2(18.1-33.2)$ & $23(17.3-36)$ & 0.905 \\
\hline Histology & & & 0.618 \\
\hline SCC & $11(50.0)$ & $37(58.7)$ & \\
\hline Non-SCC & $11(50.0)$ & $26(41.3)$ & \\
\hline Grade $^{\mathrm{a}}$ & & & 0.737 \\
\hline 1 & $3(13.6)$ & $6(9.5)$ & \\
\hline 2 & $11(50.0)$ & $33(52.4)$ & \\
\hline 3 & $7(31.8)$ & $14(22.2)$ & \\
\hline Unknown & $1(4.5)$ & $10(15.9)$ & \\
\hline LVSI $^{\mathrm{a}}$ & & & 0.444 \\
\hline Negative & $10(45.5)$ & $35(55.5)$ & \\
\hline Positive & $11(50.0)$ & $24(38.1)$ & \\
\hline Unknown & $1(4.5)$ & $4(6.3)$ & \\
\hline Maximum tumor diameter (mm) & $20.5(1-57)$ & $19(1-65)$ & 0.634 \\
\hline Tumor diameter & & & 0.464 \\
\hline$\leq 20 \mathrm{~mm}$ & $10(45.5)$ & $35(55.6)$ & \\
\hline$>20 \mathrm{~mm}$ & $12(54.5)$ & $28(44.4)$ & \\
\hline Depth of stromal infiltration & $5(0.6-19)$ & $5(0.4-25)$ & 0.953 \\
\hline FIGO stage 2018 & & & 0.602 \\
\hline IA-IB & $16(72.7)$ & $40(63.5)$ & \\
\hline IIA-IIB/IIIc1p & $6(27.3)$ & $23(36.5)$ & \\
\hline Pathologic parametrial infiltration & & & 0.332 \\
\hline No & $22(100.0)$ & $57(90.5)$ & \\
\hline Yes & 0 & $6(9.5)$ & \\
\hline Pathologic lymph node metastasis & & & 0.918 \\
\hline No & $17(77.3)$ & $48(76.2)$ & \\
\hline Yes & $5(22.7)$ & $15(23.8)$ & \\
\hline Neo-adjuvant treatment & & & 0.435 \\
\hline No & $21(95.5)$ & $55(87.3)$ & \\
\hline Yes & $1(4.5)$ & $8(12.7)$ & \\
\hline Approach & & & 0.299 \\
\hline Laparotomy & $9(40.9)$ & $18(28.6)$ & \\
\hline MIS & $13(59.1)$ & $63(71.4)$ & \\
\hline SLN metastasis ${ }^{b}$ & & & 0.739 \\
\hline No & $11(73.3)$ & $49(77.8)$ & \\
\hline Yes & $4(26.7)$ & $14(22.2)$ & \\
\hline SLN metastasis ${ }^{\mathrm{b}}$ & & & 0.798 \\
\hline No & $11(73.3)$ & $49(77.8)$ & \\
\hline ITC & $1(6.7)$ & $2(3.2)$ & \\
\hline Micro & $3(20.0)$ & $10(15.9)$ & \\
\hline Macro & 0 & $2(3.2)$ & \\
\hline
\end{tabular}

$B M I$ body mass index, SCC squamous cell carcinoma, LVSI lymph-vascular space involvement, FIGO international federation of gynecology and obstetrics $S L N$ sentinel lymph node, ITC isolated tumor cell

${ }^{a}$ Analysis performed on cases with available data only

b 7 patients did not have SLN mapping therefore no information on SLN metastasis available 


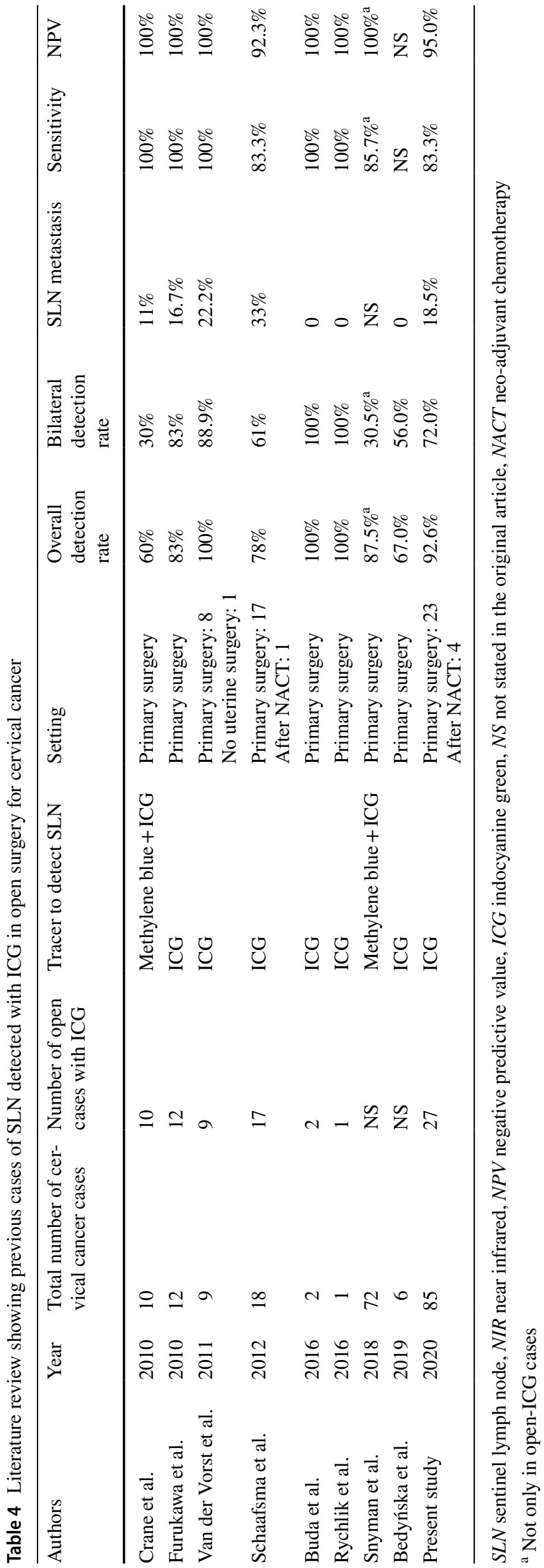

\section{Conclusion}

In conclusion, the use of ICG to detect SLN in cervical cancer treated with open surgery allows a bilateral detection, sensitivity and negative predictive value comparable to minimally invasive surgery or with open surgery using other tracers. Performance of open SLN biopsy with ICG hits the standards described for cervical cancer. The advantages of ICG compared to other tracers make it a promising tool to detect SLN in an era where open surgery and SLN biopsy has become essential part of the personalized treatment for cervical cancer.

Acknowledgements The authors thank the surgical team and the scrub nurses at Fondazione Policlinico Universitario A. Gemelli IRCCS, Rome, Italy.

Author contributions All authors contributed to the study conception and design. Material preparation, data collection and analysis were performed by Luigi Pedone Anchora, Gabriella Ferrandina, Gian Franco Zannoni, Maria Vittoria Carbone, Camilla Fedele, Elena Teodorico, Valerio Gallotta, Salvatore Gueli Alletti, Vito Chiantera, Anna Fagotti, Francesco Fanfani, Giovanni Scambia. The first draft of the manuscript was written by Nicolò Bizzarri, Francesco Fanfani and Giovanni Scambia and all authors commented on previous versions of the manuscript. All authors read and approved the final manuscript.

Funding Open access funding provided by Università Cattolica del Sacro Cuore within the CRUI-CARE Agreement. This study was not supported by funds.

Data availability All data and materials as well as software application or custom code support their published claims and comply with field standards.

\section{Compliance with ethical standards}

Conflicts of interest All authors declare no conflict of interest.

Open Access This article is licensed under a Creative Commons Attribution 4.0 International License, which permits use, sharing, adaptation, distribution and reproduction in any medium or format, as long as you give appropriate credit to the original author(s) and the source, provide a link to the Creative Commons licence, and indicate if changes were made. The images or other third party material in this article are included in the article's Creative Commons licence, unless indicated otherwise in a credit line to the material. If material is not included in the article's Creative Commons licence and your intended use is not permitted by statutory regulation or exceeds the permitted use, you will need to obtain permission directly from the copyright holder. To view a copy of this licence, visit http://creativecommons.org/licenses/by/4.0/.

\section{References}

Altgassen C, Paseka A, Urbanczyk H et al (2007) Dilution of dye improves parametrial SLN detection in patients with cervical cancer. Gynecol Oncol 105(2):329-334 
Arbyn M, Weiderpass E, Bruni L et al (2020) Estimates of incidence and mortality of cervical cancer in 2018: a worldwide analysis. Lancet Glob Health 8(2):e191-e203

Balaya V, Guani B, Magaud L et al (2019) Surgical algorithm for sentinel lymph nodes detection in early-stage cervical cancer. Int J Gynecol Cancer. https://doi.org/10.1136/ijgc-2019ESGO.3 (Nov 29 (Suppl 4) A3)

Bedyńska M, Szewczyk G, Klepacka T et al (2019) Sentinel lymph node mapping using indocyanine green in patients with uterine and cervical neoplasms: restrictions of the method. Arch Gynecol Obstet 299(5):1373-1384

Bhatla N, Aoki D, Sharma DN, Sankaranarayanan R (2018) Cancer of the cervix uteri. Int J Gynaecol Obstet 143(Suppl 2):22-36

Bizzarri N, Pedone Anchora L, Zannoni GF et al (2020) Role of onestep nucleic acid amplification (OSNA) to detect sentinel lymph node low-volume metastasis in early-stage cervical cancer. Int J Gynecol Cancer 30(3):364-371

Brisson M, Kim JJ, Canfell K et al (2020) Impact of HPV vaccination and cervical screening on cervical cancer elimination: a comparative modelling analysis in 78 low-income and lower-middleincome countries. Lancet 395(10224):575-590

Buda A, Dell'Anna T, Vecchione F, Verri D, Di Martino G, Milani R (2016) Near-infrared sentinel lymph node mapping with indocyanine green using the VITOM II ICG exoscope for open surgery for gynecologic malignancies. J Minim Invasive Gynecol 23(4):628-632

Cibula D, McCluggage WG (2019) Sentinel lymph node (SLN) concept in cervical cancer: current limitations and unanswered questions. Gynecol Oncol 152(1):202-207

Cibula D, Abu-Rustum NR, Dusek L et al (2012) Bilateral ultrastaging of sentinel lymph node in cervical cancer: lowering the falsenegative rate and improving the detection of micrometastasis. Gynecol Oncol 127(3):462-466

Cibula D, Pötter R, Planchamp F et al (2018) The European society of gynaecological oncology/European society for radiotherapy and oncology/European society of pathology guidelines for the management of patients with cervical cancer. Int J Gynecol Cancer 28(4):641-6558

ClinicalTrials. Feasibility study: conservative treatment in cervical cancer. ClinicalTrials.gov Identifier: NCT02323841. https ://clinicaltrials.gov/ct2/show/NCT02323841?term $=$ scamb ia\&draw $=2 \&$ rank $=1$. Accessed 26 Sept 2020

Crane LM, Themelis G, Pleijhuis RG et al (2011) Intraoperative multispectral fluorescence imaging for the detection of the sentinel lymph node in cervical cancer: a novel concept. Mol Imaging Biol 13(5):1043-1049

Di Martino G, Crivellaro C, De Ponti E et al (2017) Indocyanine green versus radiotracer with or without blue dye for sentinel lymph node mapping in stage $>$ IB 1 cervical cancer $(>2 \mathrm{~cm})$. J Minim Invasive Gynecol 24(6):954-959

Dostálek L, Zikan M, Fischerova D et al (2018) SLN biopsy in cervical cancer patients with tumors larger than $2 \mathrm{~cm}$ and $4 \mathrm{~cm}$. Gynecol Oncol 148(3):456-460

Dostalek L, Slama J, Fisherova D et al (2020) Impact of sentinel lymph node frozen section evaluation to avoid combined treatment in early-stage cervical cancer. Int J Gynecol Cancer 30(6):744-748

Furukawa N, Oi H, Yoshida S, Shigetomi H, Kanayama S, Kobayashi $\mathrm{H}$ (2010) The usefulness of photodynamic eye for sentinel lymph node identification in patients with cervical cancer. Tumori 96(6):936-940

Guani B, Balaya V, Magaud L, Lecuru F, Mathevet P (2020) The clinical impact of low-volume lymph nodal metastases in earlystage cervical cancer: the senticol 1 and senticol 2 trials. Cancers (Basel) 12(5):1061

Kara PP, Ayhan A, Caner B et al (2008) Sentinel lymph node detection in early stage cervical cancer: a prospective study comparing preoperative lymphoscintigraphy, intraoperative gamma probe, and blue dye. Ann Nucl Med 22(6):487-494

Kocian R, Slama J, Fischerova D et al (2020) Micrometastases in sentinel lymph nodes represent a significant negative prognostic factor in early-stage cervical cancer: a single-institutional retrospective cohort study. Cancers (Basel) 12(6):E1438

Koh WJ, Abu-Rustum NR, Bean S et al (2019) Cervical cancer version 3.2019, NCCN clinical practice guidelines in oncology. J Natl Compr Canc Netw 17(1):64-84

Marnitz S, Köhler C, Bongardt S et al (2006) Topographic distribution of sentinel lymph nodes in patients with cervical cancer. Gynecol Oncol 103(1):35-44

Nitecki R, Ramirez PT, Frumovitz M et al (2020) Survival after minimally invasive vs. open radical hysterectomy for early-stage cervical cancer: a systematic review and meta-analysis. JAMA Oncol 6(7):1-9 (published online ahead of print, 2020 Jun 11)

Papadia A, Gasparri ML, Mueller MD (2017) Are allergic reactions to indocyanine green really that uncommon? A single institution experiences. Obstet Gynecol Rep 1(2):1-2

Pedone Anchora L, Turco LC, Bizzarri N et al (2020) How to select early-stage cervical cancer patients still suitable for laparoscopic radical hysterectomy: a propensity-matched study. Ann Surg Oncol 27(6):1947-1955

Querleu D, Cibula D, Abu-Rustum NR (2017) 2017 update on the querleu-morrow classification of radical hysterectomy. Ann Surg Oncol 24(11):3406-3412

Querleu D, Cibula D, Concin N et al (2020) Laparoscopic radical hysterectomy: a European society of gynaecological oncology (ESGO) statement. Int J Gynecol Cancer 30(1):15

Ramirez PT, Frumovitz M, Pareja R et al (2018) Minimally invasive versus abdominal radical hysterectomy for cervical cancer. $\mathrm{N}$ Engl J Med 379(20):1895-1904

Robova H, Halaska MJ, Pluta M et al (2014) Oncological and pregnancy outcomes after high-dose density neoadjuvant chemotherapy and fertility-sparing surgery in cervical cancer. Gynecol Oncol 135(2):213-216

Rychlik A, Marin S, De Santiago J, Zapardiel I (2016) Utility of laparoscopic indocyanine green-guided sentinel node biopsy in open cervical cancer surgery. Int J Gynecol Cancer 26(7):1288-1289

Salvo G, Ramirez PT, Levenback CF et al (2017) Sensitivity and negative predictive value for sentinel lymph node biopsy in women with early-stage cervical cancer. Gynecol Oncol 145(1):96-101

Schaafsma BE, van der Vorst JR, Gaarenstroom KN et al (2012) Randomized comparison of near-infrared fluorescence lymphatic tracers for sentinel lymph node mapping of cervical cancer. Gynecol Oncol 127(1):126-130

Schwartz GF, Giuliano AE, Veronesi U (2002) Proceedings of the consensus conference on the role of sentinel lymph node biopsy in carcinoma of the breast, April 19-22, 2001, Philadelphia, Pennsylvania. Cancer 94:2542-2551

Silva LB, Silva-Filho AL, Traiman P et al (2005) Sentinel node detection in cervical cancer with $(99 \mathrm{~m})$ Tc-phytate. Gynecol Oncol 97(2):588-595

Slama J, Dundr P, Dusek L et al (2012) Sentinel lymph node status in patients with locally advanced cervical cancers and impact of neoadjuvant chemotherapy. Gynecol Oncol 125(2):303-306

Snyman LC, Bryant EP, Wethmar EI et al (2018) Use of a sentinel lymph node biopsy algorithm in a south African population of patients with cervical cancer and high prevalence of human immunodeficiency virus infection. Int J Gynecol Cancer 28(7):1432-1437

Tax C, Rovers MM, de Graaf C, Zusterzeel PL, Bekkers RL (2015) The sentinel node procedure in early stage cervical cancer, taking the next step; a diagnostic review. Gynecol Oncol 139(3):559-567 
van der Vorst JR, Hutteman M, Gaarenstroom KN et al (2011) Optimization of near-infrared fluorescent sentinel lymph node mapping in cervical cancer patients. Int J Gynecol Cancer 21(8):1472-1478

Wright TC, Stoler MH, Behrens CM et al (2015) Primary cervical cancer screening with human papillomavirus: end of study results from the ATHENA study using HPV as the first-line screening test. Gynecol Oncol 136(2):189-197

Wydra D, Sawicki S, Wojtylak S, Bandurski T, Emerich J (2006) Sentinel node identification in cervical cancer patients undergoing transperitoneal radical hysterectomy: a study of 100 cases. Int J Gynecol Cancer 16(2):649-654

Publisher's Note Springer Nature remains neutral with regard to jurisdictional claims in published maps and institutional affiliations. 